\title{
Adoptive immunotherapy in patients with recurrent malignant glioma: preliminary results of using autologous whole-tumor vaccine plus granulocyte-macrophage colony-stimulating factor and adoptive transfer of anti-CD3-activated lymphocytes
}

\author{
Andrew E. Sloan, M.D., Roger Dansey, M.D., Lucia Zamorano, M.D., Dr. Med., \\ Geoffrey Barger, M.D., Caroline Hamm, M.D., Fernando Diaz, M.D., Ph.D., \\ Roy Baynes, M.D., Ph.D., ANd Gary Wood, Ph.D.
}

Departments of Neurosurgery, Internal Medicine, and Neurology, Karmanos Cancer Institute; and Wayne State University Medical School, Detroit, Michigan

\begin{abstract}
Object. This trial was designed to determine the ability of autologous whole-tumor cell vaccines to induce cellmediated immune responses in patients with recurrent malignant glioma, as well as to determine whether combining such vaccination with adoptive transfer of in vitro activated $\mathrm{T}$ lymphocytes prolongs patient survival.

Methods. Nineteen patients with recurrent malignant glioma, in whom previous external beam radiotherapy and at least one course of chemotherapy had failed were vaccinated twice with irradiated autologous whole tumor cells by using granulocyte-marcrophage colony-stimulating factor as an adjuvant. Patients then underwent leukapheresis followed by adoptive transfer of peripheral blood lymphocytes activated in vitro with anti-CD3 and interleukin-2. In vivo immune response, radiological response, clinical outcome, and survival were monitored.

Seventeen patients developed a delayed-type hypersensitivity (DTH) response to vaccination that appeared to be directed against the autologous tumor. In eight patients there was radiological evidence of a response and in five there was evidence of clinical improvement. Median survival was 12 months (range 6-28 months), and both the presence of a DTH response and the radiological response correlated with survival ( $\mathrm{p}<0.02$ and $\mathrm{p}<0.04$, respectively).

Conclusions. These preliminary results suggest that autologous whole-tumor cell vaccines induce a cell-mediated immune response, which appears to be tumor specific in most patients. Furthermore, vaccination combined with adoptive immunotherapy with in vitro activated cells may induce a radiologically demonstrated tumor response and improved survival despite a condition of advanced disease and immunosuppression resulting from previous treatment or tumor burden. Further studies of immunotherapy are warranted.
\end{abstract}

\section{KEY WORDS - adoptive immunotherapy - autologous cancer cell vaccine - glioma • granulocyte-macrophage colony-stimulating factor $\bullet$ T lymphocyte $\bullet$ malignant tumor}

Gliomas are the most common primary neoplasms of the central nervous system, accounting for more than half of the estimated 20,765 primary central nervous system tumors diagnosed each year in the United States. ${ }^{8}$ Glioblastoma multiforme and AA, which together comprise the majority of malignant gliomas, are the most common primary brain tumors in adults aged 45 to 74 years $^{8}$ and account for $27 \%$ of these. Multimodality treatmentcombined resection, radiotherapy, and chemotherapyhas long been shown to improve survival of patients with these neoplasms. ${ }^{41}$ Unfortunately, however, despite numerous trials of experimental therapy, the prognosis has not changed significantly in the last two decades: median

Abbreviations used in this paper: AA = anaplastic astrocytoma; BCG = bacillus of Calmette-Guérin; DTH = delayed-type hypersensitivity; GMB = glioblastoma multiforme; GM-CSF = granulocyte-macrophage colony-stimulating factor; IDMEM = Iscove's modification Dulbecco's minimum essential medium; IL = interleukin; $\mathrm{WBC}=$ white blood cell. survival is approximately 11 months postdiagnosis and 5 to 6 months post-tumor recurrence. ${ }^{1,4,10,13,16}$ These unfortunate results have stimulated interest in experimental approaches to the treatment of these insidious neoplasms.

Immunotherapy is a particularly appealing strategy because of its theoretical potential for tumor-specific cytotoxicity. In several clinical studies immunotherapy has been shown to mediate regression in patients with established extracranial malignancy. ${ }^{9,15,37,39,40}$ However, several theoretical concerns suggest that this approach may not be applicable to patients with gliomas. First, the brain is believed to be an immunologically privileged region due to the blood-brain barrier, lack of lymphatic drainage, and diminished expression of major histocompatability complex antigens. ${ }^{28,32}$ Second, gliomas are known to produce immunosuppressive substances such as transforming growth factor- $\beta$, prostaglandin E2, and IL-10. ${ }^{7,25,26,31}$ Lastly, the devastating consequences of brain inflammatory responses in viral encephalitis, multiple sclerosis, and experimental autoimmune encephalitis suggest the possibil- 
ity that an immune response in the brain may be poorly tolerated. ${ }^{43}$ Nonetheless, the observation that lymphocytes typically infiltrating these neoplasms are tumor specific and that active cellular immunotherapy leads to prolonged survival or cure in several animal models us to investigate the efficacy of immunotherapy in patients harboring malignant gliomas. ${ }^{14,18-21,23,24,29,30,33,35,36,38,42}$

In Phase I clinical trials of combined active and adoptive immunotherapy with autologous tumor in which BCG was used as an adjuvant, investigators have demonstrated the safety and feasibility of this approach in patients with recurrent $\mathrm{AA}$ and GBM. ${ }^{22,44}$ They found that large numbers of activated $\mathrm{T}$ lymphocytes could be produced from autologous peripheral blood by stimulating with anti-CD3 and IL-2, and the lymphocytes could be reinfused with minimal toxicity. ${ }^{44}$ Furthermore, delayed recurrence and prolonged survival in some patients suggested the clinical efficacy of this approach. ${ }^{44}$ However, BCG appeared to be a poor adjuvant for glioma, inducing in vivo immune response as evidenced by DTH in only $11 \%$ of patients. ${ }^{22}$ The recent identification of GM-CSF as a potent but nonimmunogenic adjuvant suggested that efficacy may be improved by substituting GM-CSF for BCG. ${ }^{12,13,26}$ The current trial was designed to assess the ability of an autologous whole-tumor vaccine combined with GM-CSF to induce immune responses against astrocytoma. It was further designed to determine whether combining such vaccination with adoptive transfer of anti-CD3-stimulated peripheral blood T cells would limit tumor recurrence and prolong patient survival.

\section{CLINICAL MATERIAL AND METHODS}

\section{Protocol Inclusion Criteria}

Patients reported in this ongoing study were enrolled in either of two protocols approved by the institutional review board of the Karmanos Cancer Center and Wayne State University between October 1, 1997, and March 31, 2000. The two studies were nearly identical except that one included patients with several types of malignant tumors. When this protocol expired, the second was written specifically for patients with malignant glioma. Patients with other types of tumors treated using the earlier protocol will be reported elsewhere.

Eligible patients were adults with histologically confirmed diagnoses of Grade III or IV malignant glioma that had recurred after standard treatment with external beam radiotherapy and at least one course of chemotherapy. Additional eligibility criteria included: a resection of at least $90 \%$ of the enhancing neoplasm; a Karnofsky Performance Scale score of 60 or greater; ability to be weaned from steroid medication postoperatively; a WBC count greater than $3.5 \times 10^{3} / \mu \mathrm{l}$; a platelet count greater than $100 \times 10^{3} / \mu \mathrm{l}$ normal electrolyte activity; and no chemotherapeutic agents within 30 days of first vaccination. Exclusion criteria included multifocal or brainstem gliomas; concurrent neoplastic disease; known immunosuppressive condition; and a life expectancy of less than 3 months. Written informed consent was obtained from each patient, and the treatment parameters were approved by the Food and Drug Administration.

\section{Patient Characteristics}

During the 30-month period of this ongoing study, 19 eligible patients were treated with combined active and adoptive immunotherapy in which autologous wholetumor vaccine plus GM-CSF was used. Patient characteristics are summarized in Table 1. Participants were predominately male (male/female ratio 15:4), with a median age of 50 years, a mean of 52.3 years, and a median Karnofsky Performance Scale Score of 70. Two patients 
harbored recurrent AA, one had recurrent gliosarcoma, and the remaining patients harbored GBM. All recurrent tumors were histologically documented to be at least $2 \mathrm{~cm}$ in largest diameter; and a combination of modalities including external beam radiotherapy and at least two courses of one or more chemotherapeutic regimens had failed in all patients. Three patients also underwent brachytherapy in which permanent ${ }^{125} \mathrm{I}$ implants were placed, and six were treated with gamma knife surgery at some time prior to entering the study.

\section{Vaccination Procedure}

Patients underwent stereotactic volumetric resection in which image-guided techniques were used; the goal was to resect as much of the enhancing tumor as feasible without inducing a deficit. Fresh tumor specimens obtained stereotactically from the center region of the neoplasm were transported in sterile saline to a dedicated tissue culture hood. Necrotic tumor, connective tissue, and blood clots were mechanically removed, and the tissue was minced with scissors and suspended in IDMEM containing type I collagenase $(1.0 \mathrm{mg} / \mathrm{ml})$ and Dispase $(5.0 \mathrm{mg} /$ $\mathrm{ml})$. Complete digestion was achieved within 1.5 to 2 hours at $37^{\circ} \mathrm{C}$ in a baffled tissue digestion flask. The cells were suspended in IDMEM supplemented with 5\% human $\mathrm{AB}^{+}$serum (tissue culture medium) and counted. Cells were then irradiated (5000 Rads) and stored frozen at $-70^{\circ} \mathrm{C}$ in IDMEM supplemented in $20 \%$ serum and $10 \%$ dimethyl sulfoxide. All cell preparations were sterile and greater than $80 \%$ viable. In selected patients, a small specimen of autologous scalp was also processed identically to the vaccine and used as a negative control.

At the time of vaccination, $2 \times 10^{7}$ tumor cells were resuspended in $1 \mathrm{ml} 500 \mu \mathrm{g}$ GM-CSF. The mixture was divided into four equal $0.25-\mathrm{ml}$ aliquots and injected intradermally into right and left upper chest and right and left groins, giving rise to small wheals. On the following four days, GM-CSF $(125 \mu \mathrm{g})$ was injected into each of the aforementioned sites. An identical vaccination was delivered 2 weeks later.

\section{Testing for Delayed-Type Hypersensitivity}

At the time of the second vaccination, patients were tested for DTH reaction, an in vivo measure of the cellmediated immune response. This was accomplished by injecting $10^{7}$ cells in $0.25 \mathrm{ml}$ of serum-free IDMEM without GM-CSG intradermally at a location distant from the immunization sites and marked with indelible ink. The maximum diameter of the resultant wheal was then measured with a caliper 24 hours later. A positive response was defined as a raised, indurated wheal $10 \mathrm{~mm}$ or more in diameter at the injection site when examined 24 hours later. In selected patients, negative controls for DTH responses were also tested for reactivity by injecting a preparation of cells derived from autologous scalp $\left(10^{7}\right.$ cells $)$, $0.25 \mathrm{ml}$ culture medium (IDMEM + 20\% autologous serum), or $125 \mu \mathrm{g}$ GM-CSF.

\section{Activation of T Lymphocytes}

Two weeks after the second immunization, mononuclear WBCs were isolated from nonmobilized peripheral blood using a cell separator. Approximately, 2 to $8 \times 10^{10}$
WBCs were obtained from each of two leukapheresis procedures that were performed 1 day apart. Plasma $(300 \mathrm{ml})$ also was collected to be used to prepare autologous serum for the culture medium. White blood cells were cultured for 48 hours in IDMEM with autologous serum (culture medium) and Orthoclone anti-CD3 in tissue culture flasks at $37^{\circ} \mathrm{C}$ and $5 \% \mathrm{CO}$. Interleukin-2 was then added, and cells were cultured for an additional 4 to 5 days. After reaching maximum density, cells were pooled and harvested into infusion bags by using a cell harvester system. Cell count and viability were determined, and morphological analysis was performed. Cells then were reinfused into patients. In the patients in cases 11 to 19 (Table 1), cells were frozen while testing for microbial and fungal contamination was performed to ensure sterility prior to reinfusion.

\section{Adoptive Cell Transfer}

After thawing, cells were resuspended and evaluated for viability by using trypan blue exclusion. Patients underwent intravenous reinfusion of 0.5 to $5 \times 10^{11}$ sterile, endotoxin-free cells over a 6-hour period and were observed for an additional 90 minutes.

\section{Toxicity and Response Criteria}

Toxicity was monitored and graded according to National Cancer Institute Common Toxicity Criterion. Radiological response was assessed at the time at which the activated lymphocytes were reinfused and thereafter at 3month intervals. A complete response was defined as disappearance of all clinical and radiological evidence of tumor; partial response as at least a $25 \%$ decrease in the sum of the cross-sectional area of the tumor; and progressive disease was defined as the appearance of a new lesion or an increase in the cross-sectional area by more than $25 \%$. Stable disease was defined as a change too small to quantify as either partial response or progressive disease. Clinical response was assessed at 3, 6, 9, and 12 months and graded improved if tumor-related symptoms such as headache or seizures improved or if neurological examination demonstrated objective improvement. Worsening symptoms or neurological findings were categorized as evidence of progressive disease. Cases that did not fit definitions of "improved" or "progressive disease" were defined as "stable."

\section{Statistical Analysis}

Survival data were compared using the Wilcoxon logrank test. Integral responses were compared using the two-tailed Fisher exact test. Statistical significance was set at the level of $\mathrm{p}<0.05$.

\section{Sources of Supplies and Equipment}

We obtained the IDMEM from Worthington Biochemical Co. (Lakewood, NJ), and Dispase from Sigma Chemical Co. (St. Louis, MO). The tissue digestion flasks were purchased from Wheaton Glass Co. (Ringoes, NJ). The GM-CSF (Sargramostim) was acquired from Immunex Corporation (Seattle, WA). Cobe (Lakewood, NJ) manufactured the Spectra cell separator used to isolate WBCs. We procured the Orthoclone and anti-CD3 from Ortho 
A. E. Sloan, et al.

Pharmaceuticals (Raritan, NJ) and the IL-4 from Chiron Corporation (Emeryville, CA). The cell harvester system was manufactured by Baxter/Fenwall (Deerfield, IL).

\section{RESULTS}

\section{Vaccination Procedure}

All patients underwent vaccination without incident. Four patients (Cases 2, 5, 7, and 10) had induration at the site of vaccination with swelling and erythema of up to 5 $\mathrm{mm}$ in diameter. There was no evidence of tumor growth at the vaccination site in any patient throughout the duration of the follow-up period.

\section{In Vitro Lymphocyte Stimulation}

High levels of cellular differentiation and proliferation occurred in cultures obtained from 18 of 19 patients. Granulocytes typically disappeared during the anti-CD3 culture period. Some monocytes adhered to the surface of the plastic flasks during the anti-CD3 stimulation. The majority of the monocytes also disappeared during culture. As lymphocytes proliferated, the aggregates became progressively smaller until the final population had become predominately $\mathrm{T}$ lymphocytes. Cells obtained in the patient in case 13 showed a minimal proliferative response to in vitro stimulation, as well as a higher than normal rate of cell death. In this patient clinical and radiologically demonstrated disease progression were observed between vaccination and leukapheresis, necessitating a pulse of dexamethasone just prior to leukapheresis, which was continued for 5 days. In addition, previous treatment with three different chemotherapy regimens had induced some degree of bone marrow suppression such that on day of vaccination, the WBC count was only $3.5 \times 10^{3} / \mu 1$.

\section{Associated Toxicity}

Patients developed transient symptoms of low-grade fever (nine patients), chills (seven patients), and muscle spasm (seven patients) during and immediately after reinfusion; such symptoms were consistent with National Cancer Institute Common Toxicity Criterion Grade I response. The other patients did not experience any adverse effects. There were no episodes of Grades II to IV toxicity observed during this treatment protocol.

\section{In Vivo Evidence of Immune Response}

Four patients developed a DTH response to their first vaccination. This suggests that some tumor antigen was recognized as "foreign" by their immune system prior to the vaccination protocol or that they responded to some of the compound used in the processing of the vaccine. Seventeen of the 19 patients developed a DTH response to autologous tumor (Table 2). In the four patients who responded to their initial vaccination there was an enhanced response to the second vaccination. In the two patients (Cases 8 and 13) in whom vaccination elicited no response, previous multiple courses of chemotherapeutic regimens (two or three, respectively) had failed and had evidence of bone marrow suppression with low WBC counts $\left(4.1 \times 10^{3} \mu \mathrm{l}\right.$ and $3.5 \times 10^{3} \mu \mathrm{l}$, respectively) had
TABLE 2

Clinical outcome in 19 patients with recurrent malignant glioma*

\begin{tabular}{cccccc}
\hline \hline $\begin{array}{c}\text { Case } \\
\text { No. }\end{array}$ & DTH & $\begin{array}{c}\text { Radiological } \\
\text { Response }\end{array}$ & $\begin{array}{c}\text { Clinical } \\
\text { Response } \\
\text { at 3 Mos. }\end{array}$ & $\begin{array}{c}\text { Survival } \\
\text { Status }\end{array}$ & $\begin{array}{c}\text { Postop } \\
\text { Survival } \\
\text { (mos) }\end{array}$ \\
\hline 1 & yes & SD & SD & dead & 12 \\
2 & yes & PR & SD & dead & 12 \\
3 & yes & PR & imp & alive & 28 \\
4 & yes & SD & SD & dead & 13 \\
5 & yes & PD & PD & dead & 6 \\
6 & yes & PR & SD & dead & 12 \\
7 & yes & PR & SD & dead & 24 \\
8 & no & SD & SD & dead & 11 \\
9 & yes & SD & SD & dead & 8 \\
10 & yes & CR & imp & alive & 17 \\
11 & yes & PR & SD & alive & 13 \\
12 & yes & PR & imp & alive & 14 \\
13 & no & PD & PD & dead & 7 \\
14 & yes & PR & SD & alive & 13 \\
15 & yes & SD & SD & dead & 11 \\
16 & yes & SD & SD & alive & 11 \\
17 & yes & SD & imp & alive & 7 \\
18 & yes & SD & SD & alive & 7 \\
19 & yes & SD & imp & alive & 7 \\
\hline
\end{tabular}

* CR = complete response; imp = improved; $\mathrm{PD}=$ progressive disease; $\mathrm{PR}=$ partial response; $\mathrm{SD}=$ stable disease.

been observed prior to starting vaccination. Survival in both patients was below median of 12 months.

Five patients (Cases 10-12, 15, and 17) underwent additional testing to assess their immune response (Table 3). The results demonstrated that these patients had competent cell-mediated immune responses to candida, a common recall antigen to which most individuals have been exposed, and that these patients did have no evidence of an immune response to substances used in preparing the vaccine itself, such as GM-CSF or tissue culture medium (IDMEM + 10\% autologous serum).

\section{Radiological Response}

In 12 of the 19 patients there was radiological evidence of increased enhancement consistent with tumor progression between surgery for recurrent tumor and reinfusion of activated lymphocytes (data not shown). Despite active disease and rapid tumor progression, radiological evidence of response to immunotherapy was demonstrated in eight patients. The patient in Case 10 was shown to have a complete radiological response (Fig. 1). Seven patients experienced partial responses at 3 months after reinfusion of the cells, and in nine patients no significant changes were revealed radiologically (Table 2 ). In two patients (Cases 5 and 13) there was evidence of radiological and clinical progression 3 months after treatment was completed. Both suffered some degree of chemotherapyinduced bone marrow suppression, as WBCs were shown to be at the lower limit of normal $\left(3.5 \times 10^{3} \mu l\right.$ and $3.7 \times$ $10^{3} / \mu 1$, respectively). Indeed, the patient in Case 13 was restarted on a course of steroid therapy prior to leukapheresis and had no DTH response as well as a poor response to in vitro stimulation. Both patients fared poorly. Univariate analysis did not reveal an association between DTH and radiological response. 
TABLE 3

Summary of immune responses in five selected patients*

\begin{tabular}{lcll}
\hline \hline Antigen & $\begin{array}{c}\text { Reaction } \\
\text { at Initial } \\
\text { Vaccination }\end{array}$ & DTH & \multicolumn{1}{c}{$\begin{array}{l}\text { Diameter } \\
\text { of Wheal } \\
\text { (median) }\end{array}$} \\
\hline candida & 5 of 5 & ND & $4-12 \mathrm{~mm}(7 \mathrm{~mm})$ \\
GM-CSF & 0 of 5 & 0 of 5 & NA \\
tissue culture media & 0 of 5 & 0 of 4 & NA \\
vaccine+ GM - CSF & 4 of 19 & 17 of 19 & $8-28 \mathrm{~mm}(14 \mathrm{~mm})$ \\
\hline
\end{tabular}

* Tests were performed in Cases 11, 12, 15, 17, and 18. Abbreviations: $\mathrm{NA}=$ not applicable $\mathrm{ND}=$ not done.

\section{Clinical Outcome}

At time of the census 10 patients had died and nine remained alive. Nine of the deaths were due to progressive neurological deterioration associated with tumor progression. The patient in Case 9 committed suicide due to financial and marital problems despite stable radiological response and stable clinical disease. The mean survival was 12.3 months, and the median survival was 12 months. Univariate analysis revealed a positive correlation between the presence of a DTH response and survival $(\mathrm{p}<$ $0.02)$. Survival also correlated with radiological response when patients who responded to therapy (those with complete or partial response) were compared with patients who did not (those whose radiological studies demonstrated stable or progressive disease) $(p<0.04)$.

Five patients improved neurologically after immunotherapy was completed. Improved function included resolution of headache (Cases 3, 12, 17, and 18), improved speech (Cases 12 and 19), improved motor power (Cases 10 and 12), and improved sensory function (Cases 12 and 17). Twelve patients remained neurologically stable for at least the first 3 months. Two patients (Cases 5 and 13) developed progressive neurological deterioration. Both had undergone at least two previous resections and had suffered chemotherapy-induced bone marrow suppression with low WBC counts, and in the patient in Case 13 poor in vivo and in vitro immune response was shown. Both fared poorly, and their survival length was well below the median. No association between clinical outcome and DTH or radiological response was revealed by univariate analysis.

Four patients underwent additional surgery for resection of recurrent gadolinium-enhancing mass while enrolled in the study. In two patients (Cases 6 and 7) there was evidence of recurrent GBM with profuse infiltrating lymphocytes, predominately of $\mathrm{CD} 4+\mathrm{T}_{\mathrm{H}}$ isotype. It was not possible to determine whether these were transferred cells, however. The patient in Case 3 was found to have primarily necrotic tissue with few viable tumor cells or lymphocytes. The patient in Case 12 underwent radiosurgery for a recurrent tumor that developed outside the original resection bed. No histological specimen was obtained.

\section{DISCUSSION}

For immunotherpy to be effective, the immune system must both identify the tumor as foreign and mount a

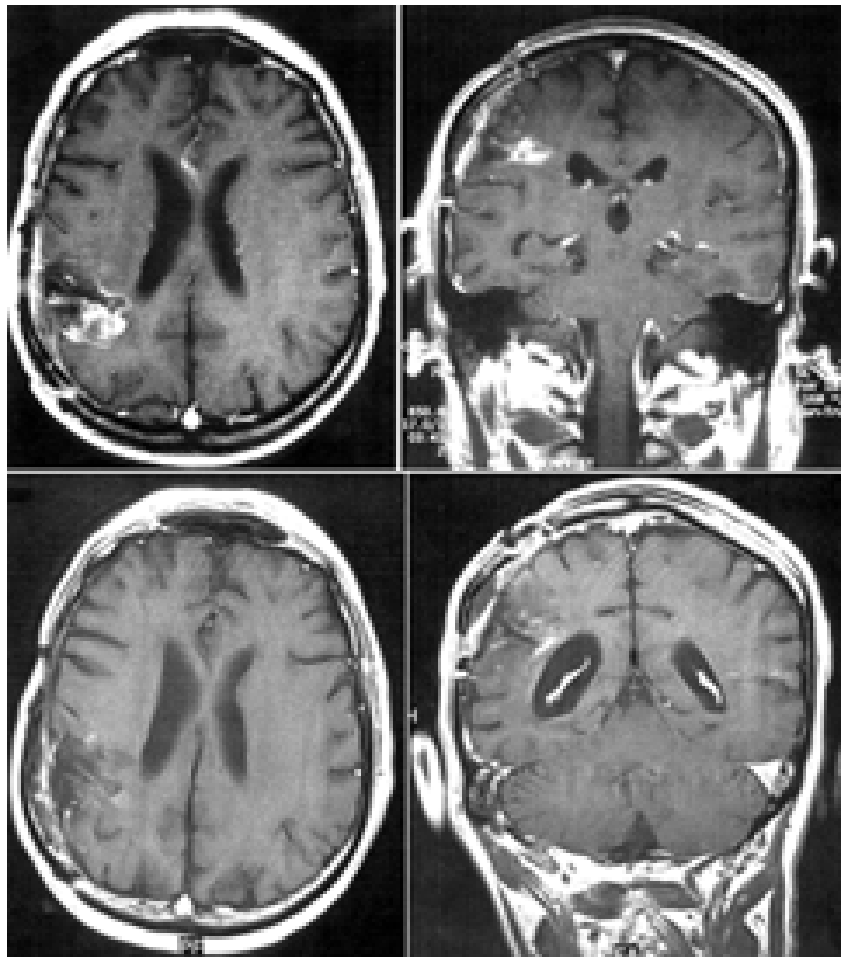

Fig. 1. Case 10. Neuroimaging studies demonstrating radiological responses. Axial (upper and lower left) and coronal (upper and lower right) magnetic resonance images obtained at time of reinfusion (upper and lower left) and 2 months following reinfusion (upper and lower right).

response to destroy it. Obstacles to immunotherapy in cases of glioma are twofold: 1) gliomas are poorly antigenic and 2) the brain's immunologically privileged nature as well as the immunosuppressive function of gliomas impair local immune responses. Whereas experimental autoimmune encephalitis, multiple sclerosis, and viral encephalitis suggest that lymphocytes can enter the brain to perform routine "immunosurveillance" activity, the paucity of major histocompatibility complex expression in the healthy brain normally induces tolerance rather than immune recognition due in part to poor antigen presentation. ${ }^{28}$ Moreover, the minimal response to even allogenic gliomas in animals and the paucity of glioma-specific antigens identified thus far in humans suggest that glioma antigens are relatively weak.

A classic strategy for improving immune recognition is vaccination with an adjuvant. This may be facilitated when it occurs in the vicinity of lymph nodes, which have high concentrations of both antigen-presenting cells and effector lymphocytes and are distant enough from primary tumor to escape any local immunosuppressive effect. Vaccination with autologous glioma and bacterial adjuvants such as BCG have facilitated the development of antitumor immune response in animal models. However, clinical studies have demonstrated that BCG was a poor adjuvant in humans, as it would frequently induce large, ulcerating lesions. ${ }^{22}$ More recently, GM-CSF has proven to be a more powerful adjuvant that is itself nonimmunogenic in humans. ${ }^{12,13,27}$ However, although vaccination 
with tumor and adjuvant effectively primes $\mathrm{T}$ lymphocytes to reject a small number of tumor cells that are later transplanted, it will not generate a sufficient number of circulating effector cells to reject an already growing neoplasm. ${ }^{9}$

Rejection of an established tumor requires large numbers of circulating effector cells. Although it may be theoretically possible to generate the required number of cells by conducting multiple, repeated vaccinations, a more practical way to generate high numbers of effector T lymphocytes is to stimulate partially activate $\mathrm{T}$ lymphocytes in vitro with anti-CD3 and IL-2 after 1 to 2 vaccinations. ${ }^{3,36,46}$ The presence of anti-CD3 activates antigenprimed $\mathrm{T}$ lymphocytes and the effect on naïve $\mathrm{T}$ cells is minimal. ${ }^{36}$ Results obtained in animal studies indicated that combining the vaccination strategy with in vitro activation could not only generate tumor-specific immune recognition but could also lead to rejection of established intracranial tumors. ${ }^{20,21,23,24,45}$

Numerous other approaches to adoptive immunotherapy for gliomas have been previously explored. Adoptive transfer of in vitro activated lymphocytes from vaccinedraining lymph nodes is a similar approach that has demonstrated efficacy in preclinical models, as well as in a small number of clinical responses in humans. ${ }^{34,36}$ However, recent evidence suggests that peripheral blood lymphocytes are more effective at homing to tumor in situ than nodal lymphocytes. ${ }^{44}$ Moreover, surgical interruption of lymphatic drainage may preclude future repeated vaccinations. Tranfection of tumors with cytokine genes has been shown to be effective in preclinical models, but because of the technical complexity of this approach, it may have little translational potential. ${ }^{14,30}$ The implantation of a cytokine-secreting pump is a simpler approach to cytokine delivery, and some efficacy has been shown in preclinical models. ${ }^{42}$ However, the mixture and timing of cytokines required to optimize immunization is not currently known and is likely to be complex. Tumor-infiltrating lymphocytes and lymphokine-activated killer cells have also been studied as possible vehicles for immunotherapy of glioma. However, both have been shown to be associated with low response rate, possibly due to poor migration to the tumor site, as well as a significant incidence of toxicity. ${ }^{4,15,37}$ In clinical trials intracavitary therapy with lymphokine-activated killer cells or allogeneic cytolytic $\mathrm{T}$ lymphocytes has been used to overcome the aforementioned problem, although its efficacy has remained modest. ${ }^{4,17}$ More recently, several groups have used dendritic cells pulsed with either tumor homogenate or tumor RNA to treat intracranial gliomas successfully in animals., ${ }^{2,18,29}$ To our knowledge, however, there have been no reports on the effectiveness of this approach in patients with malignant astrocytomas.

The current trial was designed to test two hypotheses: 1) that vaccination with autologous whole-tumor vaccine in which GM-CSF is used as an adjuvant could induce an immune response against malignant astrocytoma; and 2) that combining such vaccination with adoptive transfer of anti-CD3-stimulated peripheral blood T cells would prolong patient survival.

Analysis of our results demonstrates that, despite lack of innate immune response, intradermal vaccination with autologous whole-tumor vaccine in which GM-CSF is used as an adjuvant induces DTH reaction, a type of cellular immune response, in nearly all patients. This finding suggests that malignant gliomas are immunogenic and that, in the proper context, an antitumor immune response can be induced even in patients with advanced disease and the associated immunosuppressive consequences of increased tumor burden, chemotherapy-induced bone marrow suppression, and glioma-associated immunosuppression. The lack of DTH in our two patients with low baseline WBCs - both of whom had not benefited from multiple courses of chemotherapy with multiple agents, and one of whom had been restarted on dexamethasone, a known immunosuppressant-is consistent with this observation. Whereas lack of identified tumor-specific antigens in human gliomas makes it difficult to ascertain the specificity of this response, the absence of response to vaccination with autologous scalp, another neuro-ectodemal tissue, or to GM-CSF or tissue culture media alone suggest that this response is related to recognition of tumor antigens.

These results can also be seen to support the safety and possible clinical efficacy of combining vaccination with adoptive immunotherapy in which in vitro activated $T$ lymphocytes are used for prolonging survival. The median survival of 12 months after tumor recurrence observed in patients in this study compares favorably with that of 5 to 6 months demonstrated for patients treated with surgery alone in a recent large clinical trial, as well as to historical controls. ${ }^{1,5,6,11,16}$ Moreover, univariate analysis demonstrated in independent association between DTH and survival, and three of our four patients with evidence of innate immune response to autologous tumor also developed a radiological response and experienced prolonged survival (median 17 months). In the remaining patient, who did poorly (Case 5), three prior courses of chemotherapy had failed, and her baseline WBC count (below $4 \times 10^{3}$ ) was consistant with chemotherapy-induced marrow suppression. Univariate analysis also established a correlation between radiological response and survival. Despite theoretical concerns regarding the development of immune responses to antigens shared by tumor and normal brain and despite the presence of such cross reaction observed in several animal models, we did not find any clinical or radiological evidence of such phenomena in our series. ${ }^{43}$

The link between treatment and outcome in a small, heterogeneous series of patients is difficult, if not impossible to establish. Although the absence of correlation between DTH and clinical response may simply reflect the effect of surgical tumor debulking, the lack of any clear association between DTH and radiological response reflects our lack of understanding of this phenomenon and the need for additional study. To some extent, the observed variability in radiological response may reflect the heterogeneity in tumor biological features, immune function, and the effects of previous treatment regimens of patients in this study. Furthermore, our understanding of the radiological appearance correlates of the immune response in the brain is limited. Although one might expect diminution of the tumor, consideration of the radiological appearance of multiple sclerosis or viral encephalitis - other diseases of the brain with causes etiology - suggests the inflammatory response might actually lead to increased enhancement. Further studies will be needed to provide better under- 
standing of the relationship between antitumor immune response and radiological response.

\section{CONCLUSIONS}

Preliminary evidence suggests that adoptive immunotherapy in which autologous whole-tumor cell vaccine is combined with CM-CSF followed by adoptive transfer of CD3-activated lymphocytes is associated with evidence of tumor-specific immune response. There was also evidence of clinical and radiological responses as well as prolonged survival in patients in whom adequate baseline immune function was established. Toxicity was minimal and selflimited, and no evidence of autoimmune disease was observed. Further studies are needed to characterize better the in vivo and in vitro immune response and to identify the role of the various lymphocyte subpopulations in this response. An even more vigorous immune response would be expected in patients in whom tumors were newly diagnosed prior to commencing immunosuppressive chemotherapy, and additional studies in this patient population are warranted.

\section{Acknowledgments}

The authors acknowledge Nilam Patel, B.S., and Suzie Bredernitz, B.S., for their technical assistance, Marci Lodej, R.N., and Sandy Kugelman, R.N., for their assistance with patient care, Christina Dales, R.N.(C), and Karen Leo for their help with coordinating this study, and Jill Barnholtz-Sloan, Ph.D., for statistical support. We also wish to thank Drs. Mark Cambell, Robert Ho, and Norman Portnoy for their participation. Finally, we thank the patients and their families for their trust, cooperation, and participation in this study.

\section{References}

1. Ammaratti M, Galicich JH, Arbit E, et al: Reoperation in the treatment of recurrent intracranial malignant gliomas. Neurosurgery 21:607-614, 1987

2. Ashley DM, Faiola B, Nair S et al: Bone marrow-generated dendritic cells pulsed with tumor extract or tumor RNA induce antitumor immunity against central nervous sytem tumors. J Exp Med 186:1177-1182, 1997

3. Baldwin NG, Rice CD, Tuttle TM, et al: Ex vivo expansion of tumor-draining lymph node cells using compounds which activate intracellular signal transduction. I. Characterization and in vivo anti-tumor activity of glioma-sensitized lymphocytes. J Neurooncol 32:19-28, 1997

4. Barba D, Saris SC, Holder C, et al: Intratumoral LAK cell and interleukin-2 therapy of human gliomas. J Neurosurg 70: $175-182,1989$

5. Berger MS, Tucker A, Spence A, et al: Reoperation for glioma. Clin Neurosurg 39:172-186, 1992

6. Brem H, Piantadosi S, Burger PC, et al: Placebo-controlled trial of safety and efficacy of intraooperative controlled delivery by biodegradable polymers of chemotherapy for recurrent glioblastomas. Lancet 345:1008-1012, 1995

7. Castelli MG, Chiabrando C, Fanelli R, et al: Prostaglandin and thromboxane synthetase by human intracranial tumors. Cancer Res 49:1505-1508, 1989

8. Central Brain Tumor Registry of the United States: Year 2000 Standard Statistical Report. The Central Brain Tumor Registry of the United States. website: www.cbtrus.org

9. Chang AE, Aruga A, Cameron MJ, et al: Adoptive Immunotherapy with vaccine-primed lymph node cells secondarily activated with anti-CD3 and interleukin-2. J Clin Oncol 15: 796-807, 1997
10. Davis FG, Freels S, Grutsch J, et al: Survival rates in patients with primary malignant brain tumor stratefied by age and type: an analysis based on Surveillance, Epidemiology, and End Results (SEER) data, 1973-1991. J Neurosurg 88:1-10, 1998

11. Dirks P, Bernstein M, Muller PJ, et al: The value of reoperation for recurrent glioblastoma. Can J Surg 36:271-275, 1993

12. Disis ML, Bernard H, Shiota FM, et al: Granulocyte-macrophage colony-stimulating factor: an effective adjuvant for protein and peptide-based vaccines. Blood 88:202-210, 1996

13. Dranoff G, Jaffee E, Lazenby A, et al: Vaccination with irradiated tumor cells engineered to secrete murine GM-CSF stimulates potent, specific, and long-lasting anti-tumor immunity. Proc Natl Acad Sci USA 90:3539-3543, 1993

14. Glick RP, Lichtor T, de Zoeten E, et al: Prolongation of survival of mice with glioma treated with semmiallogeneic fibroblasts secreting interleukin-2. Neurosurgery 45:867-874, 1999

15. Goedegebuure PS, Douvill LM, Li H, et al: Adoptive immunotherapy with tumor-infiltrating lymphocytes and interleukin-2 in patients with metastatic malignant melanoma and renal cell carcinoma: a pilot study. J Clin Oncol 13:1939-1949, 1995

16. Harsh GR, Levin VA, Gutin PH, et al: Reoperation for recurrent glioblastoma and anaplastic astrocytoma. Neurosurgery 21: 615-620, 1987

17. Hayes RL, Koslow M, Heisinger EM, et al: Improved long term survival after intracavitary interleukin-2 and lymphokine activated killer cells for adults with recurrent malignant glioma. Cancer 76:840-852, 1995

18. Heimberger AB, Crotty LE, Archer GE, et al: Bone marrowderived dendritic cells pulsed with tumor homogenate induce immunity against syngeneic intracerebral glioma. J Neuroimmunol 103:16-25, 2000

19. Holladay FP, Choudhuri R Heitz T, et al: Generation of cytotoxic immune responses during the progression of a rat glioma. J Neurosurg 80:90-96, 1994

20. Holladay FP, Heitz T, Chen YL, et al: Successful treatment of malignant rat glioma with cytotoxic T lymphocytes. Neurosurgery 31:528-533, 1992

21. Holladay FP, Heitz T, Wood GW: Antitumor activity against established intracerebral gliomas exhibited by cytotoxic T lymphocytes, but not by lymphokine-activated killer cells. J Neurosurg 77:757-762, 1992

22. Holladay FP, Heitz-Turner T, Bayer WL, et al: Autologous tumor cell vaccination combined with adoptive cellular immunotherapy in patients with grade III/IV astrocytoma. J Neurooncol 27:179-189, 1996

23. Holladay FP, Lopez G, De M: Generation of cytotoxic immune responses against a rat glioma by in vivo priming and secondary in vitro stimulation with tumor cells. Neurosurgery 30: 499-505, 1992

24. Holladay FP, Wood GW: Generation of cellular immune responses against a glioma-associated antigen(s). J Neuroimmunol 44:27-32, 1993

25. Huettner C, Paulus W, Roggendorf W: Messenger RNA expression for the immunosuppressive cytokine IL-10 in human gliomas. Am J Pathol 146:317-322, 1995

26. Inge TH, Hoover SK, Susskind BM, et al: Inhibition of tumor specific cytolytic T-lymphocyte responses by transforming growth factor beta-1. Cancer Res 52:1386-1392, 1992

27. Kwak LW, Young HA, Pennington RW, et al: Vaccination with syngeneic, lymphoma-derived Ig idiotype combined with GMCSF primes mice for a protective T-cell response. Proc Natl Acad Sci USA 96:10972-10977, 1996

28. Lampson LA: Interpreting MHC class I expression and class I/II reciprocity in the CNS: reconciling divergent findings. Microsc Res Tech 32:267-285, 1995

29. Liau LM, Black KL, Prins RM, et al: Treatment of intracranial gliomas with bone marrow-derived dendritic cells pulsed with tumor antigens. J Neurosurg 90:1115-1124, 1999

30. Lichtor T, Glick RP, Kim TS, et al: Prolonged survival of mice 
with gliomas injected intracerebrally with double cytokine-secreting cells. J Neurosurg 83:1038-1044, 1995

31. Maxwell M, Galanopoulos T, Neville-Golden J, et al: Effect of the expression of transforming growth factor-beta 2 in primary human glioblastomas in immunosuppression and loss of immune surveillance. J Neurosurg 76:799-804, 1992

32. Medawar PW: Immunity to homologous grafted skin. III. The fate of skin homografts transplanted to the brain, subcutaneous tissue, and to the anterior chamber of the eye. Br J Exp Pathol 29:58-69, 1948

33. Meischer S, Whiteside TL, de Tribolete N, et al: In situ characterization, clonogenic potential, and anti-tumor cytolytic activity of T lymphocytes infiltrating human brain cancers. J Neurosurg 68:438-448, 1988

34. Plautz GE, Barnett GH, Miller DW, et al: Systemic adoptive immunotherapy of malignant gliomas. J Neurosurg 89:42-51, 1998

35. Plautz GE, Inoue M, Shu S: Defining the synergistic effects of irradiation and T-cell immunotherapy for murine intracranial tumors. Cell Immunol 171:277-284, 1996

36. Plautz GE, Toualisky JE, Shu S: Treatment of murine gliomas by adoptive transfer of ex vivo activated tumor draining lymph node cells. Cell Immunol 178:101-107, 1997

37. Rosenberg SA, Packard BS, Aebersold PM, et al: Use of tumorinfiltrating lymphocytes and interleukin-2 in the immunotherapy of patients with metastatic melanoma. A preliminary report. N Engl J Med 319:1675-1680, 1988

38. Sampson JH, Archer GE, Ashley DM, et al: Subcutaneous vaccination with irradiated, cytotkine-producing tumor cells stimulates $\mathrm{CD}^{+}$cell-mediated immunity against tumors located in the "immunologicially privileged" central nervous system. Proc Natl Acad Aci USA 93:10399-10404, 1996

39. Sussman JJ, Shu S, Sondak VK, et al: Activation of T lymphocytes for the adoptive immunotherapy of cancer. Ann Surg Oncol 1:296-306, 1994

40. Vermorken JB, Claessen AME, van Tinteren HA, et al: Active specific immunotherapy for stage II and stage III human colon cancer: a randomized trial. Lancet 353:345-350, 1999
41. Walker MD, Alexander E Jr, Hunt WE, et al: Evaluation of BCNU and/or radiotherapy in the treatment of anaplastic gliomas. A cooperative trial. J Neurosurg 49:333-343, 1978

42. Wallenfriedman MA, Conrad JA, DelaBarre L, et al: Effects of continuous localized infusion of granulocyte-macrophage colony-stimulating factor and inoculations of irradiated glioma cells on tumor regression. J Neurosurg 90:1064-1071, 1999

43. Wikstrand CJ, Bigner DD: Hyperimmunization of non-human primates with BCG-CS and cultured human glioma-derived cells. Production of reactive antisera and absence of EAE induction. J Neuroimmunol 1:249-260, 1981

44. Wood GW, Holladay FP, Turner T, et al: A pilot study of autologous cancer cell vaccination and adoptive cellular immunotherapy using anti-CD3 stimulated lymphocytes in patients with recurrent grade III/IV astrocytoma. J Neurooncol 98(2): 113-120. 2000

45. Wood GW, Turner T, Wang YY, et al: Immune rejection of intracerebral gliomas using lymphocytes from glioma-bearing rats. J Immunother 22:497-505, 1999

46. Yoshigawa H, Chang AE, Shu S: Specific adoptive immunotherapy mediated by tumor-draining lymph node cells sequentially activated with anti-CD3 and IL-2. J Immunol 147: 729-737, 1991

Manuscript received October 25, 2000.

Accepted in final form November 28, 2000.

Dr. Sloan's work on this project was supported in part by the George Kamberos Fellowship, American Brain Tumor Association; Career Development Award, American Society for Clinical Oncology; and developmental funds, Karmanos Cancer Institute and Wayne State University. Dr. Baynes's work on this project was supported by a grant from the Immunex Corporation and developmental funds, Karmanos Cancer Institute.

Address reprint requests to: Andrew E. Sloan, M.D. Department of Neurosurgery, 4160 John R. Road, Suite 930 Detroit, Michigan 48201. email: asloan@ neurosurgery.wayne.edu. 